\title{
Repair of Spontaneous Corneal Perforation in Pellucid Marginal Degeneration Using Amniotic Membrane
}

\author{
Martin Heur ${ }^{1}$, Samuel Yiu ${ }^{2 *}$ \\ ${ }^{1}$ Department of Ophthalmology, Keck School of Medicine of the University of Southern California, Los Angeles, \\ CA, USA \\ ${ }^{2}$ Department of Ophthalmology, The Wilmer Eye Institute, The John Hopkins University, Baltimore, Maryland, \\ USA \\ Email: "syiu2@jhmi.edu
}

Received 8 July 2015; accepted 27 November 2015; published 30 November 2015

Copyright (C) 2015 by authors and Scientific Research Publishing Inc.

This work is licensed under the Creative Commons Attribution International License (CC BY).

http://creativecommons.org/licenses/by/4.0/

(c) (i) Open Access

\begin{abstract}
A 47-year-old woman with a history of pellucid marginal degeneration was referred for management of hydrops and peripheral perforation of the right cornea. The initial management with cyanoacrylate tissue adhesive and bandage contact lens did not preclude aqueous leakage the next day. Amniotic membrane grafting using both a surgical graft and a bandage patch was thus performed in the operating room the following day. There was no aqueous leakage on the first postoperative day. The corneal integrity was restored with resolution of the corneal edema; and the visual acuity improved from $20 / 400$ before surgery to $20 / 40$ three months later. This case illustrates the clinical efficacy of amniotic membrane grafting as an effective alternative in the management of spontaneous corneal perforation resulted from pellucid marginal degeneration.
\end{abstract}

\section{Keywords}

Pellucid Marginal Degeneration, Corneal Perforation, Cyanoacrylate Tissue Adhesive, Amniotic Membrane

\section{Introduction}

Pellucid marginal degeneration is a non-inflammatory corneal ecstatic disorder characterized by inferior peri-

"Corresponding author.

How to cite this paper: Heur, M. and Yiu, S. (2015) Repair of Spontaneous Corneal Perforation in Pellucid Marginal Degeneration Using Amniotic Membrane. Open Journal of Ophthalmology, 5, 163-166. 
pheral thinning with rare spontaneous perforation [1]-[6]. Surgical management in the acute phase can be challenging. Here, we presented a case in which we used cryopreserved amniotic membrane as a patch graft and a Prokera device as a biologic bandage to achieve surgical repair of the perforation.

\section{Case Presentation}

A 47-year-old female with bilateral pellucid marginal degeneration was referred to the Cornea Service at the Doheny Eye Institute for management of acute hydrops and spontaneous perforation of the right cornea. The patient presented with pain and photophobia of the right eye (OD) that began the morning of the day of presentation. She denied trauma and was evaluated by an ophthalmologist, who subsequently referred her for the management of a peripheral perforation of the right cornea. The patient suffered from pellucid marginal degeneration and wore rigid gas permeable contact lenses (RGPCL). She reported difficulty tolerating the RGPCL in the same eye for two days prior to presentation. She was not on any medication, and past medical history, family history and review of systems were unremarkable.

On examination, the uncorrected visual acuity was 20/400, right eye, and RGPCL-corrected visual acuity OS was 20/40, left eye. Pupils were reactive both eyes and no afferent pupillary defect was noted. Confrontational visual fields were full and the external examination was unremarkable. Slit lamp evaluation revealed right corneal hydrops with $8 \mathrm{~mm}$ by $2 \mathrm{~mm}$ inferior peripheral epithelial defect. Aqueous leakage was noted in the area of the epithelial defect, and the anterior chamber was flat. The right lens was clear. The slit lamp examination showed inferior peripheral thinning of the left corneal stroma. The right inferior corneal perforation was initially managed with cyanoacrylate tissue adhesive and a bandage contact lens. The anterior chamber deepened slightly after the tissue adhesive application. The patient was started on moxifloxacin four times a day in the right eye.

The next day, the patient's symptoms persisted. Slit lamp examination confirmed aqueous leakage around the tissue adhesive and a shallow anterior chamber. The patient consented with surgical repair of the perforation in the operating room the following day.

Under the operating microscope, we noted an edematous cornea and shallow anterior chamber (Figure 1(a)). The cyanoacrylate tissue adhesive was debrided and the cornea denuded with $4 \%$ cocaine solution. One layer of cryopreserved amniotic membrane (Bio-Tissue, Miami, FL) was secured onto the cornea using fibrin tissue adhesive, and its corners were transfixated to the conjunctiva using interrupted 8-0 polyglactin sutures. A Prokera

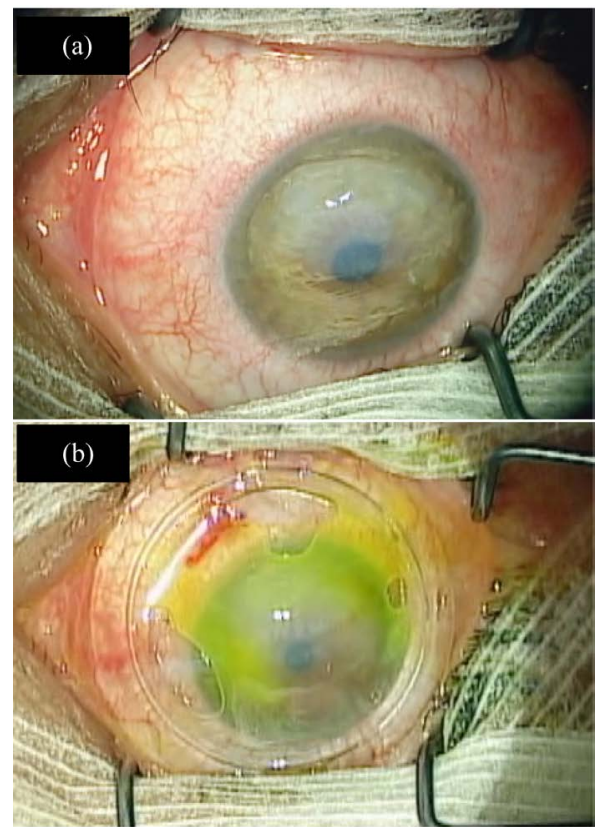

Figure 1. (a) The surgeon's view of the cornea OD shows the presence of hydrops and inferior corneal perforation prior to the surgical repair. (b) The surgeon's view of the cornea OD shows how amniotic membrane was used as a surgical graft and a bandage patch via the insertion of Prokera (Bio-Tissue, Miami, FL). 
device (Bio-Tissue, Miami, FL), amniotic membrane on a polycarbonate symblepharon ring, was then mounted on top of the amniotic membrane (Figure 1(b)). On the first post-operative day, the patient reported improvement in her symptoms. The slit lamp examination showed persistent corneal edema but a formed anterior chamber. The amniotic membrane dissolved over time. The polycarbonate ring was removed two weeks postoperatively. The patient improved gradually and she noted resolution of pain and photophobia (Figure 2(a)) on postoperative month 3 . The uncorrected visual acuity OD had improved to 20/40, and slit lamp examination revealed marked improvement in the corneal edema (Figure 2(b)).

\section{Discussion}

Pellucid marginal degeneration is a non-inflammatory corneal ecstatic disorder characterized by inferior peripheral thinning producing high against the rule astigmatism. Acute hydrops due to rupture of Descemet's membrane is a recognized sequelae in pellucid marginal degeneration. Spontaneous perforation of the cornea in pellucid marginal degeneration is exceedingly rare but had been reported [1]-[6]. Hydrops and peripheral perforation present a unique challenge for surgical management with traditional penetrating keratoplasty in the acute setting. Cyanoacrylate tissue adhesive is an alternative temporizing measure to seal the perforation and facilitate deturgescence prior to surgical repair [7]. However, as demonstrated by this present case, it is not always possible to seal a large defect. A peripheral crescentic lamellar graft combined with concurrent central penetrating keratoplasty has been proposed as a surgical technique to repair advanced cases of pellucid marginal degeneration. Technical challenge would ensue in the setting of an acute perforation with hydrops [6] [8]. Herein, we present a case in which we used cryopreserved amniotic membrane as a patch graft and a Prokera device as a temporary bandage to achieve surgical repair of the perforation. Amniotic membrane is a unique tool in the armamentarium for ocular surface reconstruction [9]-[13], as it offers several advantages in situations as illustrated in the present case. Firstly, commercial availability of amniotic membranes reduces the concern of donor cornea shortage. Secondly, amniotic membrane transplantation is technically facile, compared to a penetrating or lamellar keratoplasty. Thirdly, less surgically induced astigmatism by amniotic membrane transplantation improves visual rehabilitation. Finally, amniotic membrane grafting obviates the risk of allograft rejection that is common when corneal transplantation is performed in acute, inflamed and perforated status. Collectively, these advantages of amniotic membrane offer a viable surgical management option for cases presenting with large corneal perforation that is not amenable to initial management with cyanoacrylate tissue adhesive.

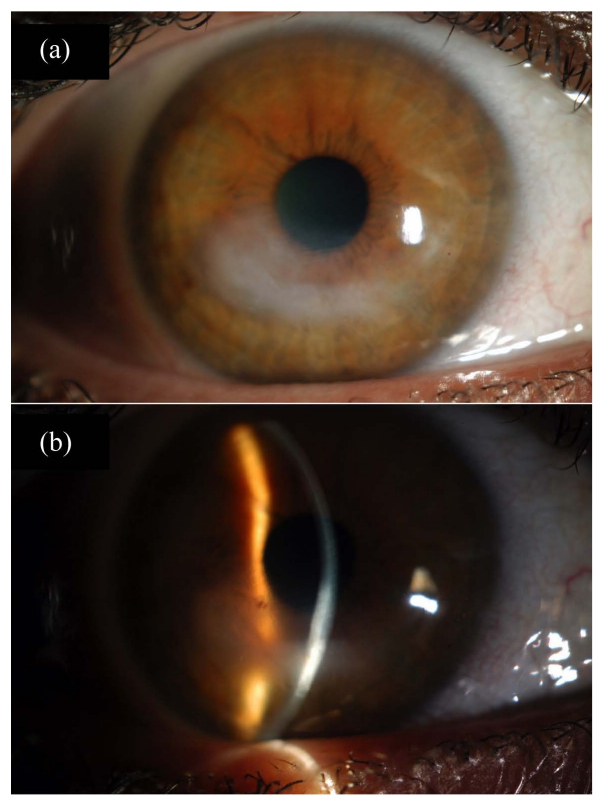

Figure 2. (a) The photograph of the cornea OD shows the restoration of corneal integrity and a marked improvement in hydrops. (b) The slit beam photograph of the cornea OD shows the marked resolution of stromal edema in the affected area. 


\section{Discussion}

Although the amniotic membrane grafting has successfully been used for treating corneal perforation caused by diverse underlying etiologies, the present case report is the first demonstration for pellucid marginal degeneration.

\section{Disclosure}

a. Financial Support: An unrestricted grant from Research to Prevent Blindness

b. Financial Disclosure(s): The authors have no financial interests in the topic of this manuscript. No conflicting relationship exists for any author.

\section{References}

[1] Akpek, E.K., Altan-Yaycioglu, R., Gottsch, J.D. and Stark, W.J. (2001) Spontaneous Corneal Perforation in a Patient with Unusual Unilateral Pellucid Marginal Degeneration. Journal of Cataract \& Refractive Surgery, 27, 1698-1700. http://dx.doi.org/10.1016/S0886-3350(01)00792-1

[2] Aldave, A.J., Mabon, M., Hollander, D.A., et al. (2003) Spontaneous Corneal Hydrops and Perforation in Keratoconus and Pellucid Marginal Degeneration. Cornea, 22, 169-174. http://dx.doi.org/10.1097/00003226-200303000-00019

[3] Lee, W.B., O’Halloran, H.S. and Grossniklaus, H.E. (2008) Pellucid Marginal Degeneration and Bilateral Corneal Perforation: Case Report and Review of the Literature. Eye \& Contact Lens, 34, 229-233. http://dx.doi.org/10.1097/ICL.0b013e318164771b

[4] Lucarelli, M.J., Gendelman, D.S. and Talamo, J.H. (1997) Hydrops and Spontaneous Perforation in Pellucid Marginal Corneal Degeneration. Cornea, 16, 232-234. http://dx.doi.org/10.1097/00003226-199703000-00018

[5] Orlin, S.E. and Sulewski, M.E. (1998) Spontaneous Corneal Perforation in Pellucid Marginal Degeneration. The CLAO Journal, 24, 186-187.

[6] Symes, R.J., Catt, C.J., Sa-ngiampornpanit, T. and Males, J.J. (2007) Corneal Perforation Associated with Pellucid Marginal Degeneration and Treatment with Crescentic Lamellar Keratoplasty: Two Case Reports. Cornea, 26, 625-628.

[7] Yiu, S.C., Thomas, P. and Nguyen, P. (2007) Review: Corneal Surface Reconstruction: Recent Advancements and Future Outlooks. Current Opinion in Ophthalmology, 18, 509-514. http://dx.doi.org/10.1097/ICU.0b013e3282f0ab33

[8] Rasheed, K. and Rabinowitz, Y.S. (2000) Surgical Treatment of Advanced Pellucid Marginal Degeneration. Ophthalmology, 107, 1836-1840. http://dx.doi.org/10.1016/S0161-6420(00)00346-8

[9] Nguyen, P. and Yiu, S.C. (2008) Ocular Surface Reconstruction: Recent Innovations, Surgical Candidate Selection and Postoperative Management. Expert Review of Ophthalmology, 3, 567-584. http://dx.doi.org/10.1586/17469899.3.5.567

[10] Kim, H.K. and Park, H.S. (2009) Fibrin Glue-Assisted Augmented Amniotic Membrane Transplantation for the Treatment of Large Noninfectious Corneal Perforations. Cornea, 28, 170-176. http://dx.doi.org/10.1097/ICO.0b013e3181861c54

[11] Ma, D.H., Wang, S.F., Su, W.Y. and Tsai, R.J. (2002) Amniotic Membrane Graft for the Management of Scleral Melting and Corneal Perforation in Recalcitrant Infectious Scleral and Corneoscleral Ulcers. Cornea, 21, 275-283. http://dx.doi.org/10.1097/00003226-200204000-00008

[12] Rodriguez-Ares, M.T., Tourino, R., Lopez-Valladares, M.J. and Gude, F. (2004) Multilayer Amniotic Membrane Transplantation in the Treatment of Corneal Perforations. Cornea, 23, 577-583. http://dx.doi.org/10.1097/01.ico.0000121709.58571.12

[13] Yildiz, E.H., Nurozler, A.B., Ozkan Aksoy, N., et al. (2008) Amniotic Membrane Transplantation: Indications and Results. European Journal of Ophthalmology, 18, 685-690. 